\title{
DNAJC3-AS1 Is Associated with Proliferation, Metastasis, and Poor Prognosis of Breast Cancer
}

\author{
Yi Zhang, ${ }^{1}$ Jing-jing Li, ${ }^{1}$ Bo Luo, ${ }^{2}$ Xiao-fei Guo, ${ }^{1}$ Jian-xin Liu, ${ }^{1}$ and Shun-shi Yang $\mathbb{D}^{1}$ \\ ${ }^{1}$ Department of Ultrasound, The Central Hospital of Wuhan, Tongji Medical College, Huazhong University of Science \\ and Technology, Wuhan, Hubei, China \\ ${ }^{2}$ Department of Pathology, The Central Hospital of Wuhan, Tongji Medical College, Huazhong University of Science \\ and Technology, Wuhan, Hubei, China
}

Correspondence should be addressed to Shun-shi Yang; jiangfengxia@zxhospital.com

Received 14 August 2021; Revised 15 October 2021; Accepted 26 October 2021; Published 3 November 2021

Academic Editor: Guo Chen

Copyright (c) 2021 Yi Zhang et al. This is an open access article distributed under the Creative Commons Attribution License, which permits unrestricted use, distribution, and reproduction in any medium, provided the original work is properly cited.

Objective. Long noncoding RNA DNAJC3-AS1 (DNAJC3-AS1) was a newly identified tumor-related lncRNA. The aim of the present study was to explore the prognostic value and diagnostic of DNAJC3-AS1 (DNAJC3-AS1) expression in breast cancer (BC) patients. Patients and Methods. The expression of DNAJC3-AS1 was detected in $170 \mathrm{BC}$ tissues and matched normal breast samples by qRT-PCR. The diagnostic value of DNAJC3-AS1 was examined by receiver-operating characteristic (ROC) assays. The correlation of DNAJC3-AS1 with clinicopathological features and prognosis was also statistically analyzed. CCK-8 assays, colony formation assays, and Transwell assays were applied to examine the potential function of DNAJC3-AS1 on tumor progression. Western blot was used to examine the expression of EMT-related proteins. Results. The expression of DNAJC3-AS1 in BC specimens was higher than that in the adjacent nontumor tissues $(p<0.01)$. Diagnostic assays revealed that DNAJC3-AS1 has considerable diagnostic accuracy, with an area under the ROC curve (AUC) of $0.7457(p<0.001)$. High DNAJC3-AS1 expression was positively associated with lymph node metastasis $(p=0.010)$ and clinical stage $(p=0.023)$. A survival study revealed that patients with high DNAJC3-AS1 expression had shorter overall survival $(p=0.0067)$ and diseasefree survival $(p<0.0001)$ than those with low DNAJC3-AS1 expression. More importantly, multivariate assays indicated that DNAJC3-AS1 was an independent prognostic factor in BC patients. Functional assays confirmed that silence of DNAJC3-AS1 distinctly suppressed the proliferation, metastasis, and EMT progress of BC cells. Conclusions. DNAJC3-AS1 may be a prognostic and diagnostic biomarker for BC patients.

\section{Introduction}

Breast cancer (BC) is the most common type of cancer and the main type of cancer that leads to the death of females around the world [1]. It was estimated that 1.65 million cases would be diagnosed with $\mathrm{BC}$ and the death toll would reach 535,800 in 2018 [2]. In China, BC, with an increasing incidence on a yearly basis, seriously threatens the health and even the life of females [3]. In recent decades, people have made a lot of efforts to find out the molecular mechanism regulating $\mathrm{BC}$ genesis. Despite the big contribution of chemotherapeutic agents to the obvious reduction of the mortality of $\mathrm{BC}$ patients, those in an advanced state still hold a poor survival because of the aggressive clinical behavior $[4,5]$. Therefore, the identification of novel molecules that are involved in the pathogenesis and progression of $\mathrm{BC}$ is essential for the improvement of $\mathrm{BC}$ diagnostic and therapy.

More than $70 \%$ of human genomes are under active transcription, while protein-coding genes only occupy $12 \%$ of human genomes [6]. Noncoding RNAs (ncRNAs) constitute most transcripts, which include long ncRNAs (lncRNAs). lncRNAs belong to a class of ncRNAs with the length over 200 nucleotides [7, 8]. IncRNAs regulate different levels of gene expression to participate in various biological processes 
including differentiation, angiogenesis, immune responses, and apoptosis [9]. More and more studies have reported the role of lncRNAs in the initiation and development of tumors as oncogenes and/or tumor suppressors [10]. Besides, the abnormal expression profiles of lncRNAs in clinical BC specimens can affect the grade of malignancy as well as the histological differentiation, exhibiting significant clinical implications for diagnosing cancer subclassification $[11,12]$. Based on these findings, lncRNAs can be potentially used to diagnose and treat tumors as a key target $[13,14]$. Thus, the identification of novel lncRNAs involved in the progression of $\mathrm{BC}$ was urgent and necessary.

lncRNA DNAJC3-AS1, located on 13q32.1, was a novel tumor-related IncRNA which was firstly demonstrated to be highly expressed in osteosarcoma and promote the proliferation and metastasis by its sense-cognate gene DNAJC3 [15]. Studies have confirmed that DNAJC3 could help cells fight against the endoplasmic reticulum stress so to suppress cellular apoptosis, thus influencing cellular biological response, such as cellular damages or programmed cell death [16]. Then, a distinct upregulation of DNAJC3-AS1 was also observed in colon cancer [17]. However, the expression pattern and function of DNAJC3-AS1 in other tumors remained largely unclear. In this study, we firstly provided evidences that DNAJC3-AS1 expression was distinctly upregulated in BC patients and predicted a poor prognosis. In addition, we confirmed that knockdown of DNAJC3-AS1 suppressed the proliferation, migration, invasion, and EMT progress of BC cells. Our findings suggested DNAJC3-AS1 as a prognostic and therapeutic target for $\mathrm{BC}$ patients.

\section{Patients and Methods}

2.1. Patients and Tissue Samples. Tumor tissues with paired adjacent normal tissues were obtained from 170 BC patients at the Central Hospital of Wuhan, Tongji Medical College, Huazhong University of Science and Technology, from July 2013 to August 2016. All those patients had not underwent radiotherapy, chemotherapy, or endocrine treatment prior to surgery. All samples, after quick-freezing, were stored at $-80^{\circ} \mathrm{C}$ for $\mathrm{qRT}-\mathrm{PCR}$. The clinical examination, together with the histopathological analysis on tissue specimens, helped to make a diagnosis. Patients' characteristics are described in Table 1. All BC patients were followed for 60 months. The study has obtained the approval from the Ethics Committee of the Central Hospital of Wuhan, Tongji Medical College, Huazhong University of Science and Technology, and obtained patients' informed consent before beginning. The study was carried out following the Helsinki Declaration.

2.2. Cell Lines and Transfection. Human BC cell lines ZR-75-1, MCF-7, BT-20, MDAMB-231, and SKBR3 and an immortalized breast epithelial cell line MCF-10A were purchased from American Type Culture Collection (ATCC, Manassas, VA, USA). All cells were cultured in RPMI-1640, supplemented with $10 \% \mathrm{FBS}$ at $37^{\circ} \mathrm{C}$ in a humidified atmosphere of $5 \%$ $\mathrm{CO}_{2}$. Small interfering RNA of DNAJC3-AS1 and the negative control siRNA were obtained from Sigma-Aldrich (USA).
TABLE 1: The correlations between DNAJC3-AS1 expression and clinicopathological factors of BC patients.

\begin{tabular}{|c|c|c|c|c|}
\hline \multirow[t]{2}{*}{ Characteristics } & \multirow[t]{2}{*}{$N$} & \multicolumn{2}{|c|}{$\begin{array}{l}\text { DNAJC3-AS1 } \\
\text { expression }\end{array}$} & \multirow[t]{2}{*}{$p$} \\
\hline & & High & Low & \\
\hline Age (years) & & & & 0.775 \\
\hline$\leq 50$ & 81 & 41 & 40 & \\
\hline$>50$ & 89 & 47 & 42 & \\
\hline Tumor size $(\mathrm{cm})$ & & & & 0.259 \\
\hline$\leq 2$ & 94 & 45 & 49 & \\
\hline$>2$ & 76 & 43 & 33 & \\
\hline Differentiation grade & & & & 0.950 \\
\hline $\mathrm{G} 1+2$ & 102 & 53 & 49 & \\
\hline G3 & 68 & 35 & 33 & \\
\hline Histological type & & & & 0.493 \\
\hline Ductal & 97 & 48 & 49 & \\
\hline Lobular & 73 & 40 & 33 & \\
\hline ER status & & & & 0.609 \\
\hline Negative & 94 & 47 & 47 & \\
\hline Positive & 76 & 41 & 35 & \\
\hline PR status & & & & 0.150 \\
\hline Negative & 94 & 44 & 50 & \\
\hline Positive & 76 & 44 & 32 & \\
\hline HER-2 status & & & & 0.397 \\
\hline Negative & 98 & 48 & 50 & \\
\hline Positive & 72 & 40 & 32 & \\
\hline Lymph node metastasis & & & & 0.010 \\
\hline Absent & 112 & 50 & 62 & \\
\hline Present & 58 & 38 & 20 & \\
\hline Clinical stage & & & & 0.023 \\
\hline $\mathrm{I}+\mathrm{II}$ & 101 & 45 & 56 & \\
\hline III & 69 & 43 & 26 & \\
\hline
\end{tabular}

According to the manufacturer's protocol, cells were transfected with Lipofectamine 2000 (Invitrogen).

2.3. Quantitative Real-Time PCR. RNAiso Plus (Takara, Suzhou, Jiangsu, China) was used to extract total RNA from cells or tissues, and HiFiScript cDNA Kit (Invitrogen, Haidian, Beijing, China) was used to reverse-transcribe the extracted total RNA into cDNA. ND-1200 NanoDrop spectrophotometer (NanoDrop, Shenzhen, Guangzhou, China) determined the concentration and purity of RNA at the absorbance at $280 \mathrm{~nm}$. The miScript Reverse Transcription together with miScript SYBR Green PCR Kit assisted in performing the real-time quantitative RT-PCR, following the protocol of manufacturers (Foster City, CA, USA). GAPDH was used as an endogenous control. The fold changes were calculated with the relative quantification $\left(2^{-\Delta \Delta \mathrm{Ct}}\right)$ method. All reactions were run in triplicate. Invitrogen (Haidian, Beijing, China) provided the primers for PCR. The primer 
sequences were as follows: DNAJC3-AS1, forward ( $5^{\prime}$-AGCG ATTGTGGAAGACCTCC- $\left.3^{\prime}\right)$ and reverse $\left(5^{\prime}\right.$-ATTTCCCCT GGTACACGGCT- $\left.3^{\prime}\right)$; GAPDH, forward $\left(5^{\prime}\right.$-GGTGA AGGTCGGAGTCAACGC- $\left.3^{\prime}\right)$ and reverse ( $5^{\prime}$-CAAAGT TGTCATGGAG-3').

2.4. Proliferation Cell Counting Kit-8 (CCK-8) Assays. CCK8 assays were conducted by the use of a CCK- 8 assay kit (Abbexa, Aimei Technology, Hubei, China). The transfected cells were seeded into culture plates, followed by the treatments of $10 \mu \mathrm{L}$ of CCK- 8 reagent. We measured absorbance at $450 \mathrm{~nm}$.

2.5. Colony Formation Assay. Cells were transfected with siDNAJC3-AS1 or si-NC, at a density of 400 cells $/ 6 \mathrm{~cm}$ dish. The above cells were trypsinized and counted. After the surviving cells developed colonies, $3.7 \%$ methanol was used to fix colonies, followed by $0.1 \%$ crystal violet. Colonies containing at least 50 cells were scored.

2.6. Transwell Migration and Invasion Assays. 24-well transwell chambers with an $8 \mu \mathrm{m}$ pore polycarbonate membrane insert were used to study the effects of DNAJC3-AS1 knockdown on metastasis ability of BC cells. $4 \times 10^{5}$ cells were loaded onto the upper compartment. To the lower compartment, $500 \mu \mathrm{L}$ medium was added. A chamber coated with Matrigel (Corning, Pudong, Shanghai, China) was applied for invasion assays. Giemsa (Solarbio, Hangzhou, Zhejiang, China) was applied for the stain of the invasive and migrative cells. A light microscope was used to count the cells.

2.7. Western Blot. Firstly, $20 \mu \mathrm{L}$ proteins were separated by $10 \%$ SDS-PAGE (Beyotime, Hangzhou. Zhejiang, China). Subsequently, $0.5 \mu \mathrm{m}$ PVDF membranes (Millipore, Hangzhou. Zhejiang, China) were applied for the transfection of the above proteins. After blocking with skim milk, the membranes were incubated overnight at $4^{\circ} \mathrm{C}$ with anti-E-cadherin, anti-N-cadherin, anti-Vimentin, and anti-GAPDH antibodies. Then membranes were incubated with a horseradish peroxidaseconjugated secondary antibody for 1 hour. Enhanced chemiluminescence was applied to detect the signal. All antibodies are purchased from Abcam (Pudong, Shanghai, China).

2.8. Statistical Analysis. Analyses were performed using GraphPad Prism (Prism 6) and R 3.6.1 software. The Student $t$-test or the chi-square test helped examine whether the difference was statistically significant between groups. Kaplan-Meier survival curves evaluated the survival, and the survival difference between groups was examined by the log-rank test. Multivariate analyses on the prognostic values were performed with the help of a Cox proportional hazard regression analysis. A $p$ value less than 0.05 was considered with statistical significance.

\section{Results}

3.1. DNAJC3-AS1 Is Upregulated in Human BC Tissues. For confirming DNAJC3-AS1 expression pattern in BC tissues, we collected 170 pairs of $\mathrm{BC}$ specimens and matched normal tissues from $170 \mathrm{BC}$ patients in our hospital and further per-

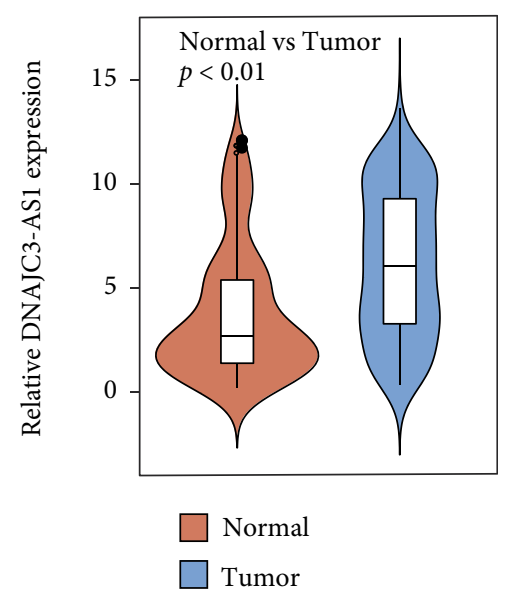

FIgURE 1: Expression of DNAJC3-AS1 is increased in BC tissues compared with noncancerous tissues through qRT-PCR $(p<0.01)$. Student's $t$-test was used for the comparison.

formed RT-PCR. As presented in Figure 1, our group observed that relative DNAJC3-AS1 levels were distinctly higher in BC tissues than those in normal breast specimens $(p<0.01)$.

3.2. The Possible Diagnostic Significance of DNAJC3-AS1 Expression for $B C$ Patients. Given the distinct overexpression of DNAJC3-AS1 in BC patients, we wondered whether DNAJC3-AS1 could have a diagnostic value. According to the ROC assays, our group observed that high DNAJC3AS1 expression had an AUC value of 0.7457 (95\% CI: 0.6934 to 0.7980 ) for BC (Figure 2). The ideal cut-off value was 5.55. Our data suggested that DNAJC3-AS1 may be an indicator for the diagnosis of $\mathrm{BC}$ patients.

3.3. Association of DNAJC3-AS1 Expression with Clinicopathological Features of BC Patients. Previous studies have reported that several lncRNAs can assist in regulating the clinical progression of various tumors. Thus, the possible clinical significance exhibited by DNAJC3-AS1 expression in $\mathrm{BC}$ patients was explored. The mean expression levels of DNAJC3-AS1 (8.36) were taken into account for dividing patients with BC into a group with a high expression level and a group with a low expression level. Table 1 listed such relations. High DNAJC3-AS1 expression was observed to lead to positive lymph node metastasis $(p=0.010)$ as well as advanced clinical stage $(p=0.023)$. Nevertheless, DNAJC3AS1 expression exhibited no obvious relation to other clinicopathological characteristics (all $p>0.05$ ).

3.4. Prognostic Value of DNAJC3-AS1 Expressions for BC Patients. In recent years, more and more studies have reported the potential of lncRNAs used as novel prognostic biomarkers. Then, associations between the DNAJC3-AS1 expression and five-year survivals were evaluated by Kaplan-Meier survival assays. Importantly, we observed that patients whose DNAJC3-AS1 expression was high exhibited a shorter OS ( $p=0.0067$; Figure 3 ) and disease-free survival (DFS, $p<0.0001$; Figure 4 ) as compared with the DNAJC3- 


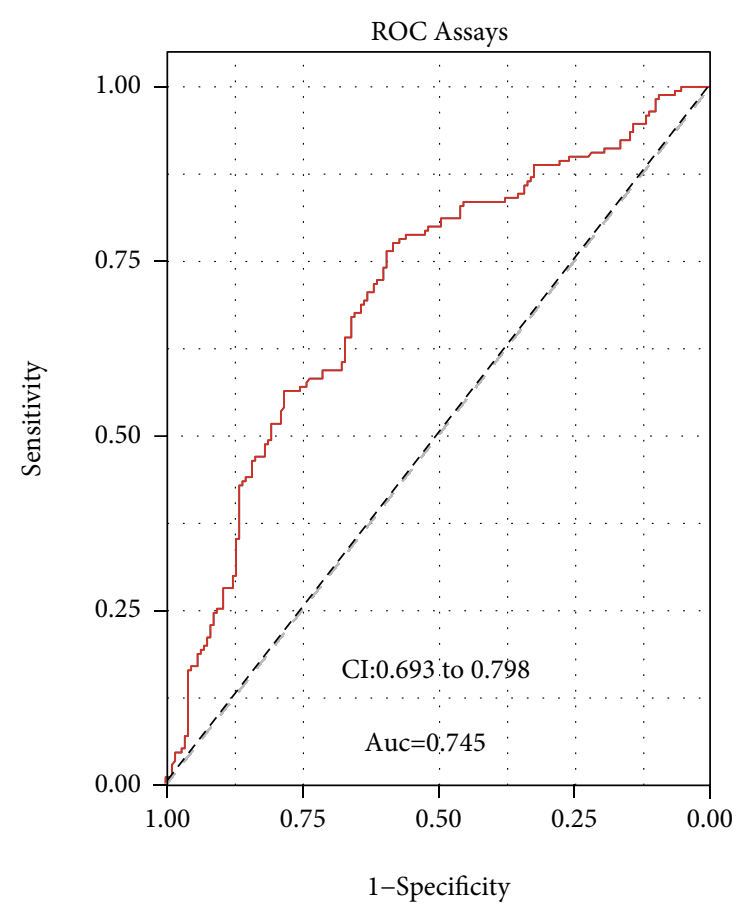

FIgURE 2: ROC curve for diagnostic value of DNAJC3-AS1 in BC patients.

AS1-low group. Moreover, multivariate assays suggested that high DNAJC3-AS1 expression was independently associated with OS (HR $=2.956,95 \% \mathrm{CI}: 1.285-4.892, p=0.003)$ and DFS ( $\mathrm{HR}=3.228,95 \% \mathrm{CI}: 1.328-5.218, p=0.001)$. Our finding revealed that increased DNAJC3-AS1 expression may be a biomarker of unfavorable outcomes for BC patients (Table 2).

3.5. Silence of DNAJC3-AS1 Promoted BC Progression. To study the functions of DNAJC3-AS1 in BC, we firstly performed RT-PCR to examine the expression of DNAJC3AS1 in several BC cells. As shown in Figure 5(a), we found that DNAJC3-AS1 expression was distinctly increased in five BC cell lines (MCF-7, BT-20, ZR-75-1, MDAMB-231, and SKBR3) compared with MCF-10A cells. siRNAs against different DNAJC3-AS1 were performed, which effectively knocked down DNAJC3-AS1 expression level in MCF-7 and BT-20 cells (Figure 5(b)). Further CCK-8 assays and colony formation assay confirmed that knockdown of DNAJC3-AS1 distinctly suppressed the proliferation of MCF-7 and BT-20 cells (Figures 5(c) and 5(d)). Furthermore, we explored whether DNAJC3-AS1 was involved in cell metastasis in MCF-7 and BT-20 cells. Transwell migration assays suggested that DNAJC3-AS1 silence distinctly decreased migration abilities in MCF-7 and BT-20 cells (Figure 6(a)). Applying invasion assays, our group reported that the number of invaded cells was distinctly reduced in the DNAJC3-AS1 knockdown cells (Figure 6(b)). Moreover, we conducted Western blot assays to detect the expressions of EMT-associated proteins after DNAJC3-AS1 silence.
Figure 6(c) indicated that knockdown of DNAJC3-AS1 distinctly promoted the expressions of E-cadherin while inhibiting the expressions of $\mathrm{N}$-cadherin and Vimentin in both MCF-7 and BT-20 cells.

\section{Discussion}

$\mathrm{BC}$, one of the most common malignant tumors, is becoming the most threatened killer of women health in the world [5]. Different patients have obviously different survival time. As the gene expression and cytology present heterogeneity, coordinating high-efficient therapeutic strategies suitable for all patients faces some difficulty $[18,19]$. The application of sensitive diagnostic and prognostic biomarkers for $\mathrm{BC}$ patients was very important for the clinical outcome of BC. Up to date, several clinical factors have been widely used in clinical detection of BC patients, and novel potential biomarkers have been also developed, such as dysregulated genes involved in tumor progression, methylation, and various ncRNAs $[20,21]$. The study paid attention to identifying novel BC-related lncRNAs.

Recently, more and more researchers have paid attention to the ability of lncRNAs to remarkably regulate the transcription process of genes. In BC, several lncRNAs have been functionally identified. For instance, lncRNA SNHG3, an overexpressed lncRNA in BC, promote BC cell proliferation and invasion via modulating miR-384/HDGF axis [22]. LINC01121, a newly identified tumor-related factor, was reported to be highly expressed in both BC specimens and the cell lines and exhibit a tumor-promotive role by promoting $\mathrm{BC}$ cell proliferation and invasion via regulating miRNA-150-5p/HMGA2 [23]. These findings suggested several functional lncRNAs as potential tumor suppressors or oncogenes in $\mathrm{BC}$, highlighting their clinical application used as novel diagnostic and prognostic biomarkers for BC. In recent years, a new lncRNA, DNAJC3-AS1, attracted our attention. In osteosarcoma, DNAJC3-AS1 expression was found to be distinctly increased. Functional assays revealed that DNAJC3-AS1 knockdown suppressed the proliferation and invasion via sense-cognate gene DNAJC3 [15]. Then, Han and his group reported that DNAJC3-AS1 expression presented a distinct upregulation in colon cancer, and its knockdown suppressed the migration and EMT progress of the colon cancer cell via miRNA-214-3p/LIVIN axis [17]. These findings revealed that DNAJC3-AS1 is a potential oncogene in tumors. However, whether DNAJC3-AS1 was dysregulated in $\mathrm{BC}$ and its clinical significance in tumor patients, including $\mathrm{BC}$, has not been investigated.

In this study, we firstly found an increase in the DNAJC3AS1 expression in BC patients, which was consistent with its overexpression in colon cancer and osteosarcoma. Then, ROC assays revealed that high DNAJC3-AS1 expression showed a great diagnostic value $(\mathrm{AUC}=0.745$; sensitivity, $82.39 \%-100 \%$; specificity, $73.24 \%-93 \%)$ in BC patients, suggesting DNAJC3-AS1 as a potential factor. Moreover, clinical research revealed that increased DNAJC3-AS1 expressions may result in advanced clinical stage, indicating that it may contribute to the clinical progress of BC. In a survival study, we observed that patients whose DNAJC3-AS1 expression is 


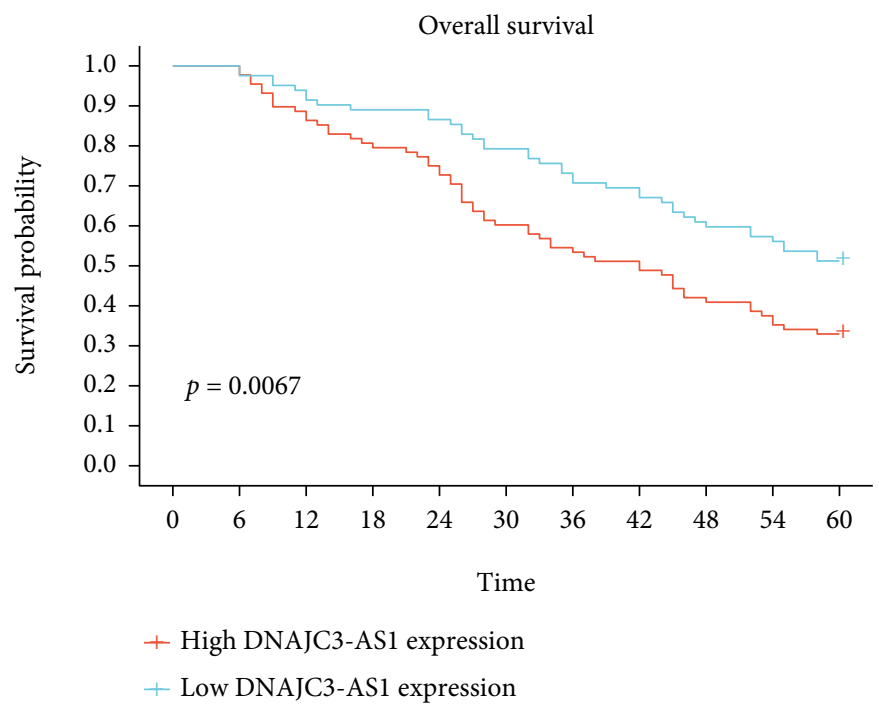

Figure 3: Kaplan-Meier assays for the overall survival in relation to DNAJC3-AS1 expression level in 170 patients with BC.

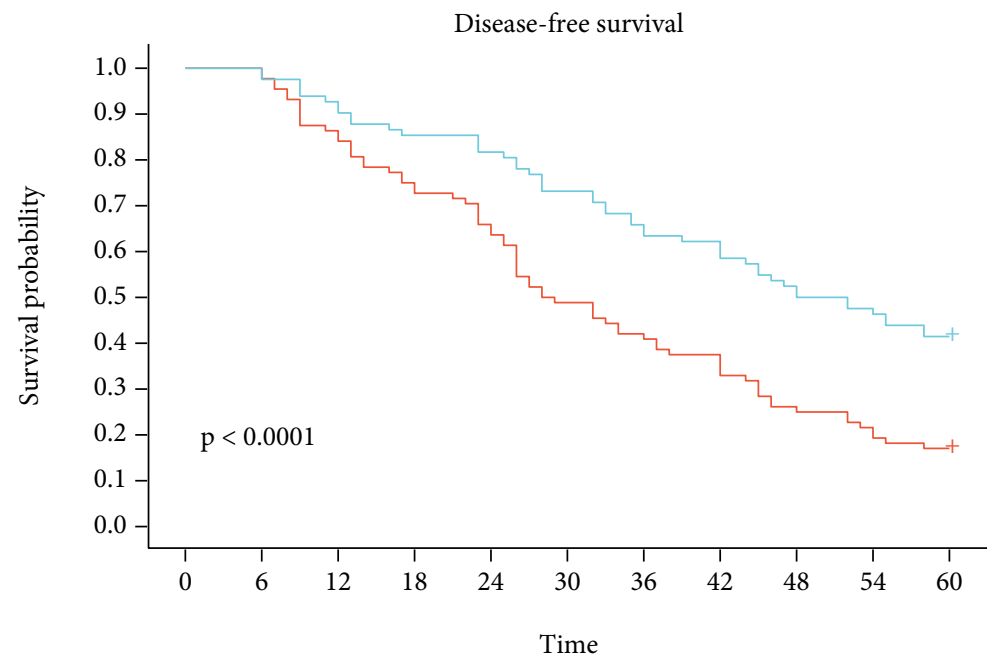

+ High DNAJC3-AS1 expression

Figure 4: Kaplan-Meier assays for the disease-free survival in relation to DNAJC3-AS1 expression level in 170 patients with BC.

TABLE 2: Multivariate survival analysis of overall survival and disease-free survival in $170 \mathrm{BC}$ patients.

\begin{tabular}{|c|c|c|c|c|c|c|}
\hline \multirow{2}{*}{ Variables } & \multicolumn{3}{|c|}{ Overall survival } & \multicolumn{3}{|c|}{ Disease-free survival } \\
\hline & $\mathrm{RR}$ & $95 \% \mathrm{CI}$ & $p$ & $\mathrm{RR}$ & $95 \% \mathrm{CI}$ & $p$ \\
\hline Age & 0.895 & $0.472-1.665$ & 0.217 & 0.938 & $0.623-1.894$ & 0.185 \\
\hline Tumor size & 1.217 & $0.774-2.184$ & 0.137 & 1.443 & $0.582-2.331$ & 0.118 \\
\hline Differentiation grade & 1.341 & $0.675-2.211$ & 0.176 & 0.986 & $0.776-1.978$ & 0.328 \\
\hline Histological type & 1.544 & 0.784-2.019 & 0.132 & 1.492 & $0.998-1.987$ & 0.247 \\
\hline ER status & 0.897 & $0.472-1.896$ & 0.337 & 1.127 & $0.632-2.018$ & 0.132 \\
\hline PR status & 1.137 & $0.663-2.123$ & 0.216 & 1.328 & $0.853-2.289$ & 0.137 \\
\hline HER-2 status & 0.986 & $0.527-1.897$ & 0.238 & 1.132 & $0.773-2.218$ & 0.198 \\
\hline Lymph node metastasis & 3.254 & $1.327-6.237$ & 0.001 & 3.582 & $1.498-7.382$ & 0.001 \\
\hline Clinical stage & 3.018 & $1.227-4.786$ & 0.008 & 3.237 & $1.426-5.334$ & 0.002 \\
\hline DNAJC3-AS1 expression & 2.956 & $1.285-4.892$ & 0.003 & 3.228 & $1.328-5.218$ & 0.001 \\
\hline
\end{tabular}




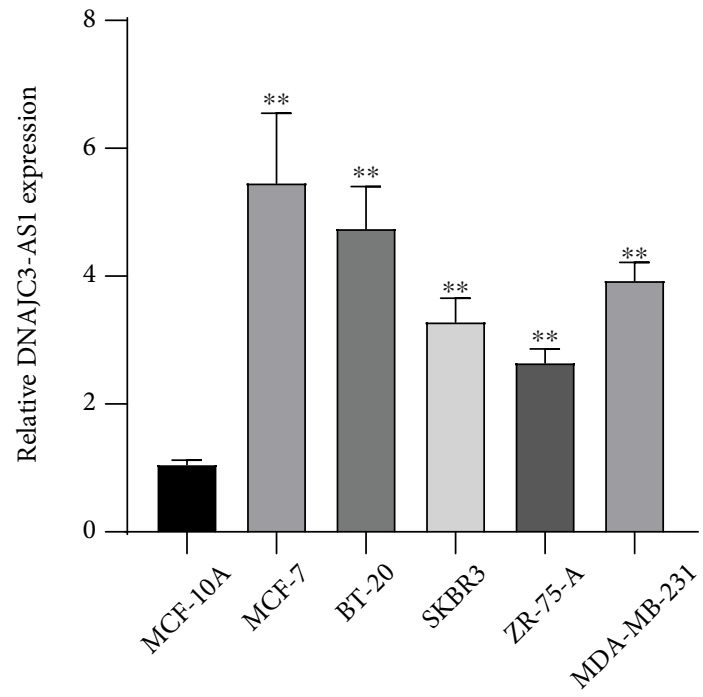

(a)

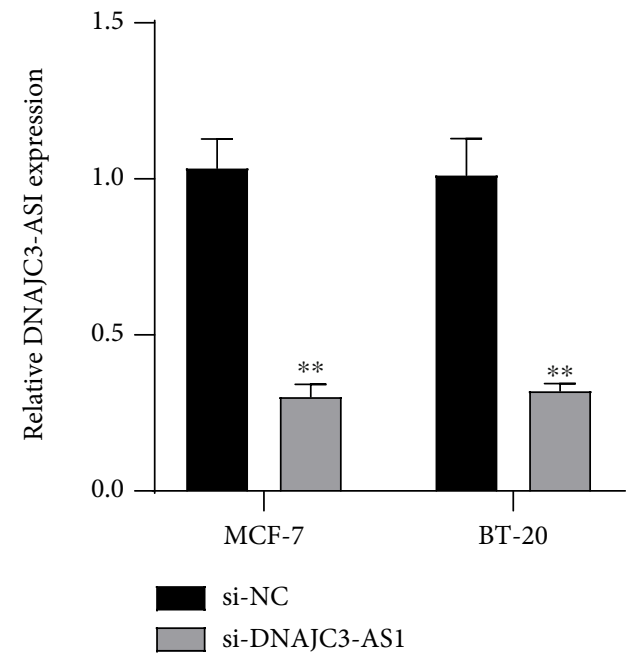

(b)
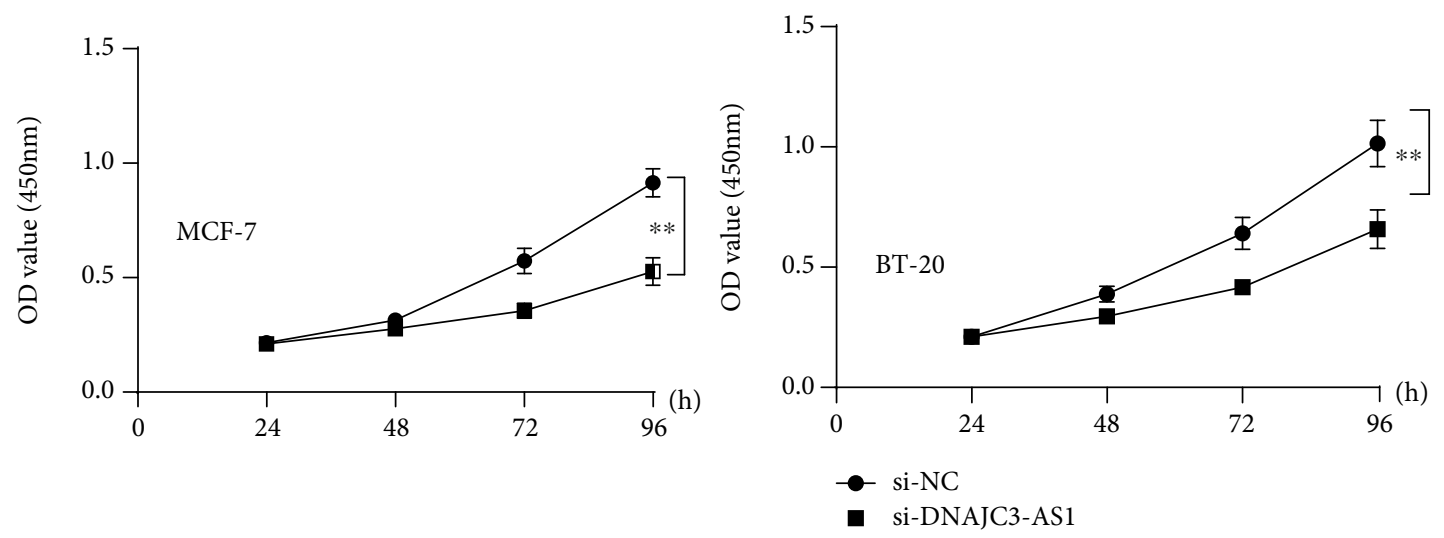

(c)
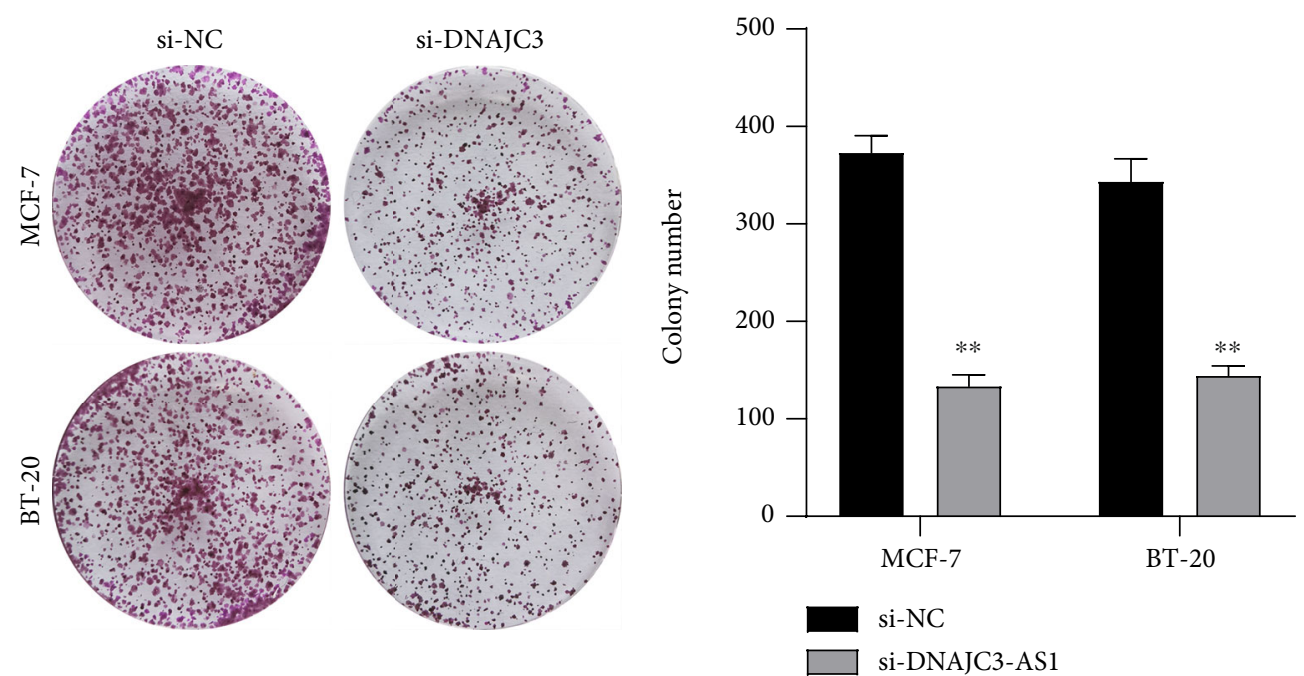

(d)

FIGURE 5: Knockdown of DNAJC3-AS1 suppressed the proliferation of BC cells. (a) The relative DNAJC3-AS1 expression was detected in five BC cells compared to MCF-10A. (b) DNAJC3-AS1 was silenced by si-DNAJC3-AS1 in MCF-7 and BT-20 cells. (c, d) CCK-8 and colony formation assays of BC cells with silencing of DNAJC3-AS1. Differences between two groups were analyzed by $t$-test. ${ }^{* *} p<0.01$. 


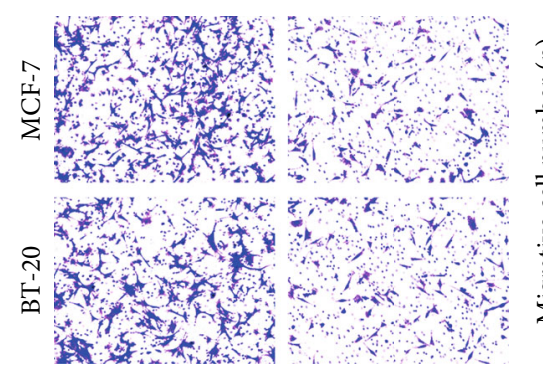

(a)
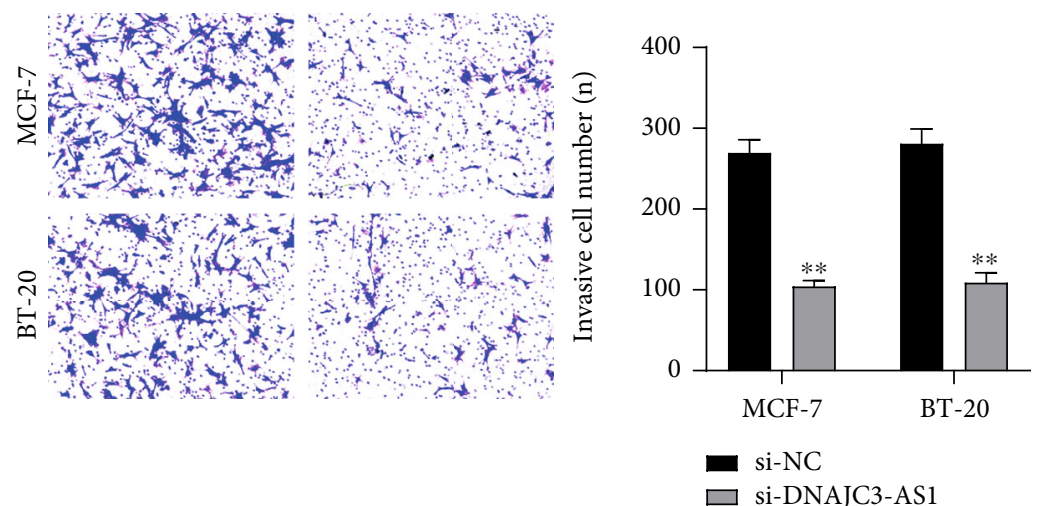

(b)

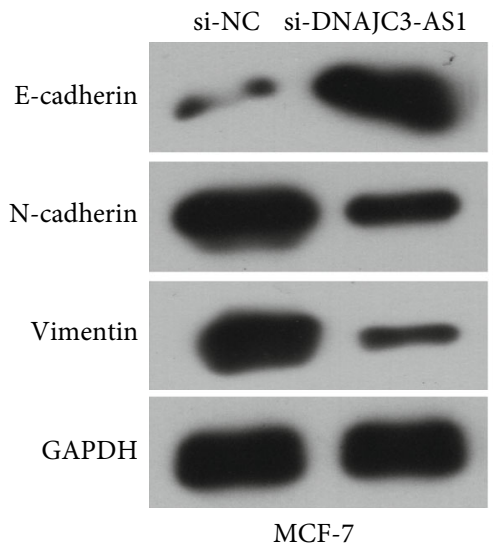

si-NC si-DNAJC3-AS1

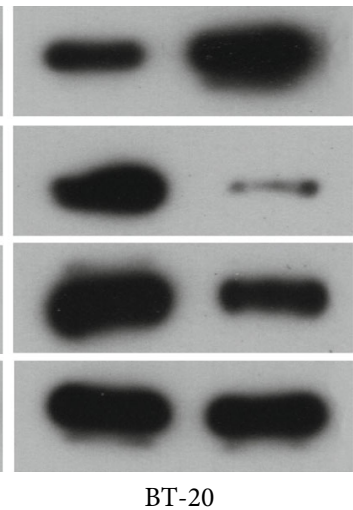

(c)

FIgURE 6: DNAJC3-AS1 knockdown inhibited the metastasis of BC cells. (a) After transfection with indicated siRNAs, migrative abilities of MCF-7 and BT-20 cells were determined using Transwell assays. (b) DNAJC3-AS1 knockdown inhibited the invasive abilities of MCF-7 and BT-20 cells. (c) The expressions of Vimentin, N-cadherin, and E-cadherin in transfected MCF-7 and BT-20 cells were detected by Western blot. Differences between 2 groups were analyzed by $t$-test. ${ }^{* *} p<0.01$.

higher exhibited a relatively shorter OS and DFS, which was confirmed by analyzing the survival of 170 patients with five-year follow-up. More importantly, multivariate assays confirmed that DNAJC3-AS1 expression could be used to independently predict the BC prognosis regarding the 5-year OS and DFS. Finally, we confirmed that knockdown of DNAJC3-AS1 distinctly suppressed the proliferation, migration, invasion, and EMT progress of BC cells. Thus, our findings firstly provided clinical evidence that DNAJC3-AS1 expression was distinctly upregulated in BC and may be used as a novel biomarker and therapeutic target for $\mathrm{BC}$ patients. However, large clinical trials are needed to further demonstrate the above results due to the small sample size in our cohort.

\section{Conclusions}

We firstly showed that the expression profile of DNAJC3AS1, an oncogenic lncRNA, might serve as a prognostic and diagnostic marker in patients with BC. Further studies are needed to examine the mechanisms underlying the oncogenic function of DNAJC3-AS1 for this malignancy.

\section{Data Availability}

The data used to support the findings of the present study are available from the corresponding author upon reasonable request. 


\section{Conflicts of Interest}

The authors declare that no conflict of interest exists in the paper.

\section{Authors' Contributions}

Yi Zhang and Jing-jing Li contributed equally to this work.

\section{Acknowledgments}

This research was supported by the funds for Youth Project of the Health Planning Committee of Hubei, China (Grant No. WJ2019H365).

\section{References}

[1] R. L. Siegel, K. D. Miller, and A. Jemal, "Cancer statistics, 2018," CA: a Cancer Journal for Clinicians, vol. 68, no. 1, pp. 7-30, 2018.

[2] C. B. Matsen and L. A. Neumayer, "Breast Cancer," JAMA Surgery, vol. 148, no. 10, pp. 971-979, 2013.

[3] L. Fan, K. Strasser-Weippl, J. J. Li et al., "Breast cancer in China," The Lancet Oncology, vol. 15, no. 7, pp. e279-e289, 2014.

[4] S. Becker, "Breast cancer in pregnancy: a brief clinical review," Best Practice \& Research. Clinical Obstetrics \& Gynaecology, vol. 33, pp. 79-85, 2016.

[5] B. Wormann, "Breast cancer: basics, screening, diagnostics and treatment," Medizinische Monatsschrift für Pharmazeuten, vol. 40, pp. 55-64, 2017.

[6] T. R. Mercer, M. E. Dinger, and J. S. Mattick, "Long noncoding RNAs: insights into functions," Nature Reviews Genetics, vol. 10, pp. 155-159, 2009.

[7] J. W. Wei, K. Huang, C. Yang, and C. S. Kang, "Non-coding RNAs as regulators in epigenetics," Oncology Reports, vol. 37, no. 1, pp. 3-9, 2017.

[8] F. Kanwal and C. Lu, "A review on native and denaturing purification methods for non-coding RNA (ncRNA)," Journal of Chromatography. B, Analytical Technologies in the Biomedical and Life Sciences, vol. 1120, pp. 71-79, 2019.

[9] G. Nigita, G. P. Marceca, L. Tomasello et al., "ncRNA editing: functional characterization and computational resources," Computational Biology of Non-Coding RNA, vol. 2019, pp. 133-174, 2019.

[10] J. R. Prensner and A. M. Chinnaiyan, "The emergence of lncRNAs in cancer biology," Cancer Discovery, vol. 1, pp. 391-407, 2011.

[11] C. M. Klinge, "Non-coding RNAs in breast cancer: intracellular and intercellular communication," Noncoding RNA, vol. 4, no. 4, p. 40, 2018.

[12] Y. Liu, S. Sharma, and K. Watabe, "Roles of lncRNA in breast cancer," Frontiers in Bioscience, vol. 7, pp. 94-108, 2015.

[13] L. Lorenzi, F. Avila Cobos, A. Decock et al., "Long noncoding RNA expression profiling in cancer: challenges and opportunities," Genes, Chromosomes \& Cancer, vol. 58, no. 4, pp. 191199, 2019.

[14] G. Yang, X. Lu, and L. Yuan, "LncRNA: a link between RNA and cancer," Biochimica et Biophysica Acta, vol. 1839, no. 11, pp. 1097-1109, 2014.
[15] R. Liang, Z. Liu, Z. Chen et al., "Long noncoding RNA DNAJC3-AS1 promotes osteosarcoma progression via its sense-cognate gene DNAJC3," Cancer Medicine, vol. 8, pp. 761-772, 2019.

[16] X. Zhang, G. Li, X. Yang et al., "Identification of a DnaJC3 gene in Apis cerana cerana and its involvement in various stress responses," Pesticide Biochemistry and Physiology, vol. 160, pp. 171-180, 2019.

[17] B. Han, Y. Ge, J. Cui, and B. Liu, "Down-regulation of lncRNA DNAJC3-AS1 inhibits colon cancer via regulating miR-2143 p/LIVIN axis," Bioengineered, vol. 11, no. 1, pp. 524-535, 2020.

[18] D. Smith, K. Thomson, C. Bambra, and A. Todd, “The breast cancer paradox: a systematic review of the association between area-level deprivation and breast cancer screening uptake in Europe," Cancer Epidemiology, vol. 60, pp. 77-85, 2019.

[19] M. E. Spei, E. Samoli, F. Bravi, C. La Vecchia, C. Bamia, and V. Benetou, "Physical activity in breast cancer survivors: A systematic review and meta- analysis on overall and breast cancer survival," Breast, vol. 44, pp. 144-152, 2019.

[20] O. Weaver and J. W. T. Leung, "Biomarkers and imaging of breast cancer," AJR. American Journal of Roentgenology, vol. 210, no. 2, pp. 271-278, 2018.

[21] B. Weigelt, J. L. Peterse, and L. J. Van't Veer, "Breast cancer metastasis: markers and models," Nature Reviews Cancer, vol. 5, no. 8, pp. 591-602, 2005.

[22] Q. Ma, X. Qi, X. Lin, L. Li, L. Chen, and W. Hu, "LncRNA SNHG3 promotes cell proliferation and invasion through the miR-384/hepatoma-derived growth factor axis in breast cancer," Human Cell, vol. 33, no. 1, pp. 232-242, 2020.

[23] Z. Wang, P. Wang, L. Cao et al., "Long intergenic non-coding RNA 01121 promotes breast cancer cell proliferation, migration, and invasion via the miR-150-5p/HMGA2 Axis," Cancer Management and Research, vol. Volume 11, pp. 10859-10870, 2019. 